\title{
Unravelling the progressive pathophysiology of idiopathic pulmonary
} fibrosis

\author{
Andreas Günther*,\#, Martina Korfei*, Poornima Mahavadi*, Daniel von der Beck*, \\ Clemens Ruppert* and Philipp Markart*
}

ABSTRACT: Idiopathic pulmonary fibrosis (IPF) is a life-threatening condition, with a median survival of $<3$ yrs. The pathophysiology is not fully understood, but chronic injury of alveolar epithelial type II cells (AECII) is considered key.

In IPF, disturbed folding and processing of surfactant proteins and impaired DNA repair may represent underlying reasons for maladaptive endoplasmic reticulum stress responses, increased reactive oxygen species production and/or DNA damage. Excessive AECII apoptosis occurs, leading to permanently perturbed epithelial homeostasis. The role of secondary hits also becomes evident. These may aggravate the disease and result in increased epithelial turnover, exhausting the regenerative capacity of progenitors and disturbing epithelial-mesenchymal interactions. Fibroblast proliferation, transdifferentiation and matrix deposition may be mediated through various mechanisms including epithelial-mesenchymal transition, fibrocyte invasion or expansion of a local fibroblast population.

Treatment modalities aiming to attenuate epithelial injury are currently in early pre-clinical development and may reach the clinical arena in only a few years. Meanwhile, novel drugs acting on highly activated fibroblasts such as pirfenidone, an anti-fibrotic drug authorised for IPF in the European Union, or BIBF 1120, a novel triple-kinase inhibitor (blocking vascular endothelial growth factor, platelet-derived growth factor and fibroblast growth factor) currently under clinical investigation, seem to attenuate the progression of IPF.

KEYWORDS: Alveolar epithelial type II cells, endoplasmic reticulum stress, fibroblast, idiopathic pulmonary fibrosis, pathophysiology

diopathic pulmonary fibrosis (IPF) is the most common form of chronic fibrosing idiopathic interstitial pneumonia (IIP). IPF is a fatal lung disease typically affecting older adults, with an estimated prevalence of 1.6 to 1.7 per 10,000 persons, and a median survival of 2-5 yrs [1-4]. A progressive decline in lung function increasingly restricts routine physical activity of the patient $[1,5]$. While the mechanisms that result in IPF are still not fully understood, there is a strong suggestion of the involvement of epithelial alveolar cells.

Myers and KATZENSTEIN [6] suggested the role of epithelial necrosis and alveolar collapse in the pathogenesis of usual interstitial pneumonia (UIP). However, this observation failed to gain a footing against the prevailing hypothesis that the development of IPF was due to a chronic inflammatory process and the resulting belief that therapeutic intervention should be directed toward arresting the inflammatory and immune response rather than the fibrotic process [7]. In 2006, SELMAN and PARDO [8] reintroduced the topic of the potential role of epithelial cells in IPF.

IPF is now thought to arise following recurrent injury to the epithelial alveolar cells, in particular the alveolar epithelial type II cell (AECII), thus, provoking responses associated with normal tissue repair and scar formation. In the pathogenesis of IPF, however, this scarring process continues unabated [9]. Risk factors for epithelial alveolar cell injury are thought to include smoking, exposure to metal or wood dust and genetic disposition, as well as age, amongst others [2].

\section{AFFILIATIONS}

*Universities of Giessen and Marburg Lung Center, Dept of Internal

Medicine II, German Center for Lung Research, Giessen, and

\#Agaplesion Lung Clinic

Waldhof-Elgershausen, Greifenstein, Germany.

\section{CORRESPONDENCE}

A. Günther

Justus-Liebig-University

Internal Medicine

Klinikstrasse 36

Giessen

35392

Germany

E-mail: andreas.guenther@uglc.de

Received:

Feb 032012

Accepted after revision:

March 162012

\section{PROVENANCE}

Publication of this peer-reviewed

article was sponsored by InterMune International AG, Muttenz,

Switzerland (article sponsor, European Respiratory Review issue 124). 
Indeed, chronic injury of AECIIs is now increasingly accepted as a key event in IPF. Injured AECIIs become susceptible to apoptosis. KORFEI et al. [10] showed that the cell markers prosurfactant protein (SP)-C and p20 caspase-3, in stained sections of IPF lungs, revealed that $70-80 \%$ of the AECIIs demonstrated ongoing signs of apoptosis.

\section{THE ROLE OF AECII IN IIPS}

The function of the AECII in the lung has been known for many years [11-13]. These cells synthesise, secrete and recycle all components of surfactant (the active agent that covers the alveolar interface), thereby reducing surface tension and allowing breathing to take place at normal transpulmonary pressures. The surfactant produced by the AECIIs has been characterised in numerous biochemical studies of bronchoalveolar lavage and is known to be composed of $~ 90 \%$ lipid and $\sim 10 \%$ protein. The surfactant proteins SP-B and SP-C, and phospholipid dipalmitoylated phosphatidylcholine are key components of surfactant [14]. The AECIIs also produce compounds of the innate immune defence system, such as defensins, collectins (of which the SP-A and SP-D are notable) and lysozyme, which all contribute towards the prevention of infections at a very low-recognition level. SP-A and SP-D, for example, are able to bind to the surface of various pathogens, thus facilitating their removal by alveolar macrophages [15-19]. The AECII cells also play a role in regulating the fluid balance in the lung, as well as having self-renewal characteristics (stem

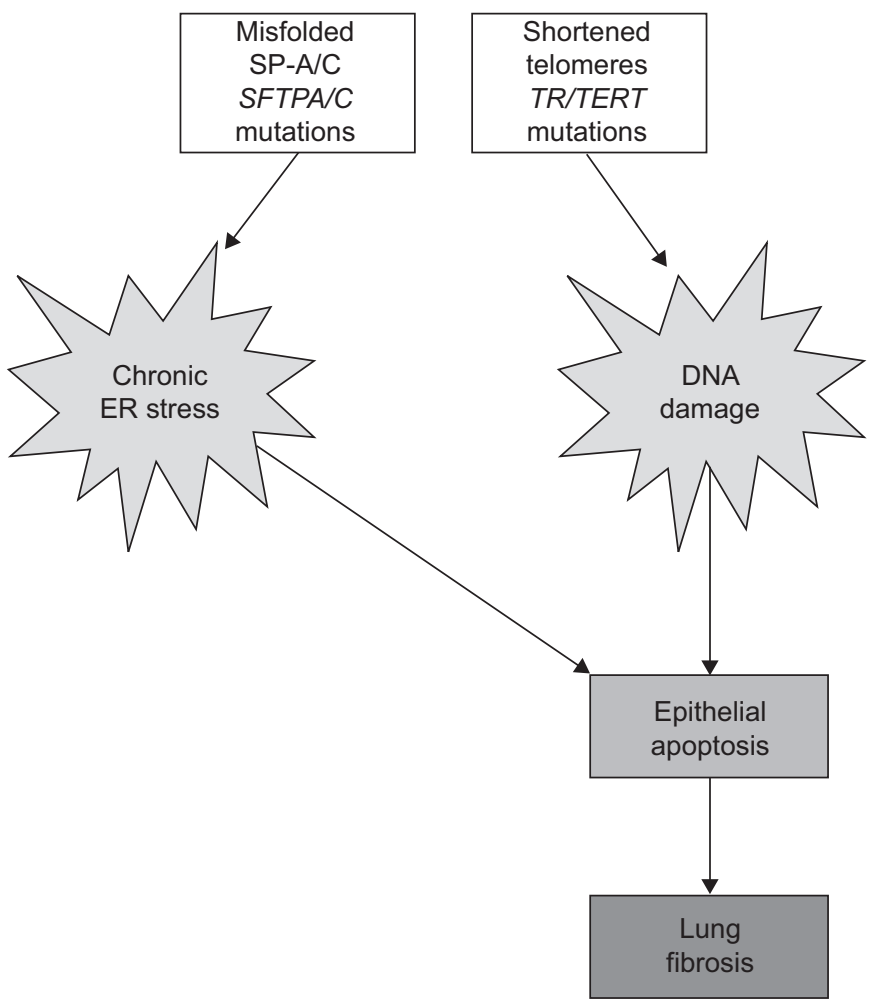

FIGURE 1. Possible mechanisms of famillial idiopathic interstitial pneumonia leading to lung fibrosis involves apoptosis caused mainly through chronic endoplasmic reticulum (ER) stress. Telomere mutations may primarily cause DNA damage once telomeres have been substantially shortened. SP-A/C: surfactant protein A/C; SFTPA/C: surfactant protein A/C gene; TR: telomerase RNA-component; TERT: telomerase reverse transcriptase. cell-like or progenitor cell) $[20,21]$. Indeed, the AECIIs have a high potential to proliferate $[14,22,23]$ and to transdifferentiate into alveolar epithelial type I cells [24].

The question that arises is why, with the capabilities of renewal and self-regulation possessed by the AECIIs, are they chronically injured in IPF? To help understand possible answers to this question, an important observation originating from familial forms of IPF and also nonspecific interstitial pneumonia is worthy of consideration. It has been found that in these families there are mutations in the surfactant proteins SP-A and SP-C, as well as in the telomeres, which appear to be associated with chronic AECII injury and apoptosis [25-30]. These mutations of the surfactant proteins seem to cause apoptosis largely through chronic stress of the endoplasmic reticulum (ER), which is the site of protein synthesis of the AECIIs. It is thought that the telomere mutations primarily cause DNA damage once the telomeres have been substantially shortened (fig. 1) [30].

At present, more than 30 different mutations of SP-C have been reported. This protein was the first to be deciphered in familial forms of IIP, and all, or most, of the mutations have been found

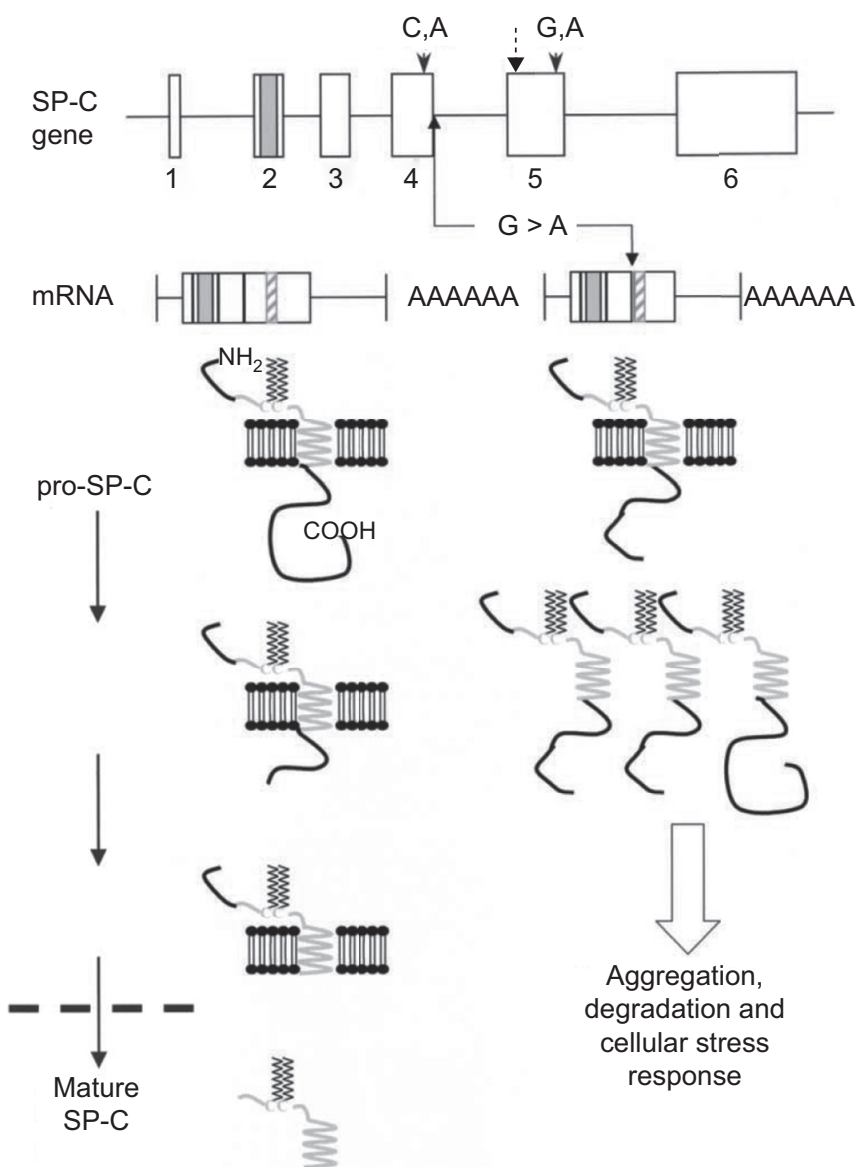

FIGURE 2. Surfactant protein (SP)-C mutations lead to protein misfolding Pathomechanism of c.460+1G $>$ A mutation, leading to alternate splicing of the SP$\mathrm{C}$ mRNA and deletion of exon 4 , and resultant production of a defective proprotein foreshortened by 37 amino acids. The mutated proSP-C protein cannot be folded and processed to its mature form, and so accumulates with the healthy SP-C proprotein. The deposition of unfolded aggregated surfactant proteins seems to cause chronic alveolar epithelial type II cell injury and eventually lung fibrosis [26]. 


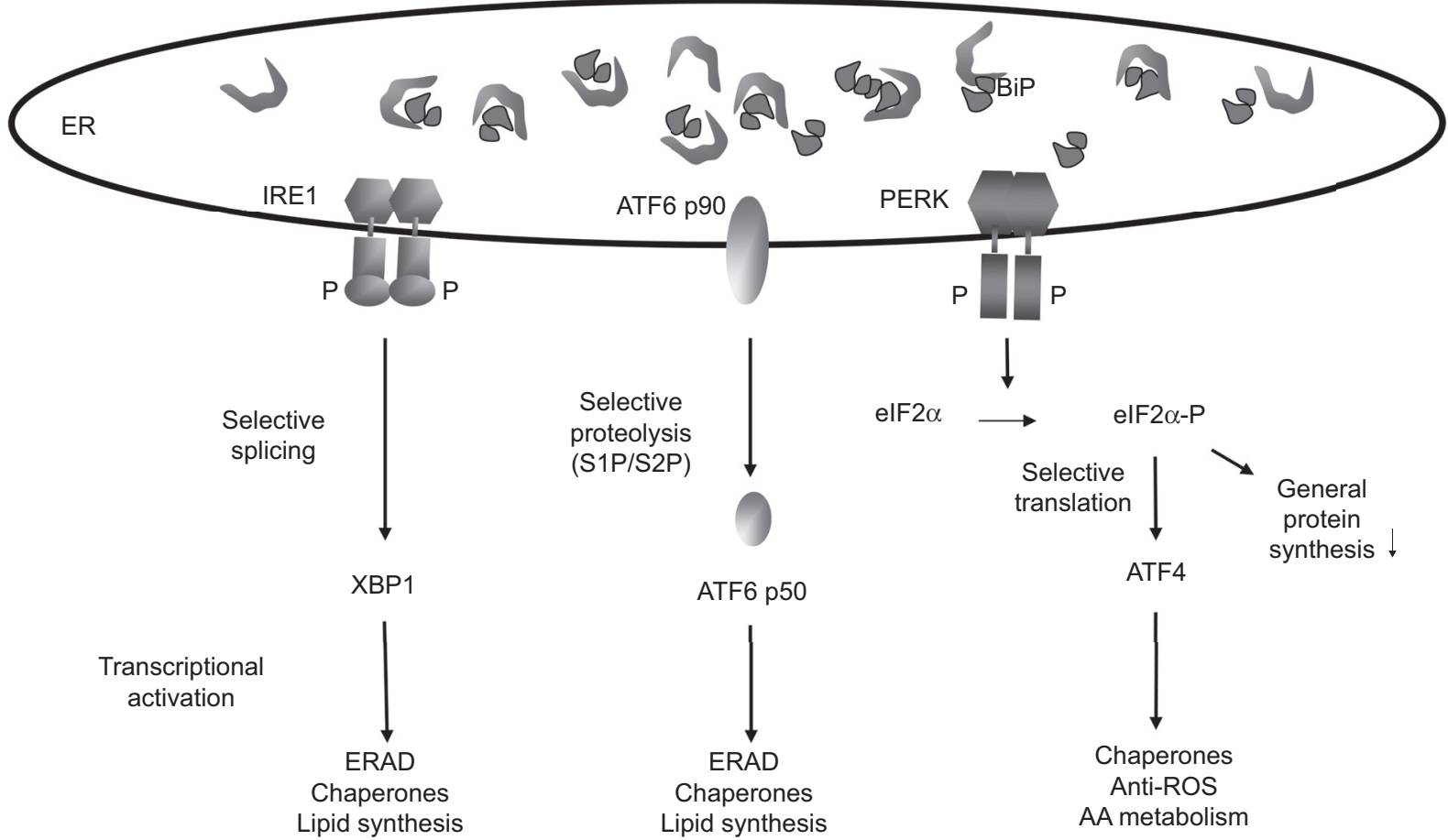

FIGURE 3. The cyctoprotective endoplasmic reticulum (ER) stress mechanism [32]. BiP: immunoglobulin heavy chain-binding protein / 78 kDa glucose-regulated protein; IRE1: inositol-requiring protein 1; ATF: activating transcription factor; PERK: protein kinase R-like endoplasmic reticulum kinase; elF: elongation initiation factor: XBP1: X-box binding protein 1; ERAD: ER-associated protein degradation; ROS: reactive oxygen species.

a)

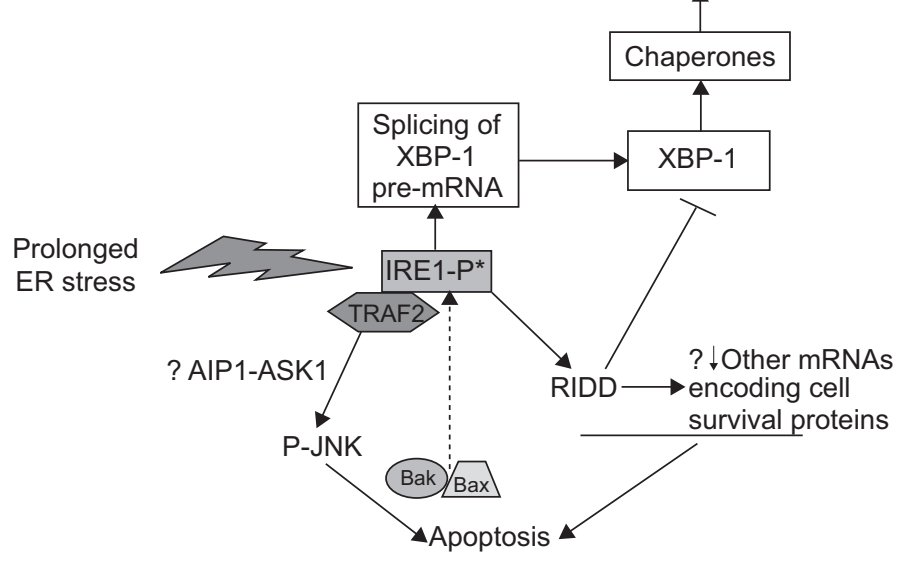

b)

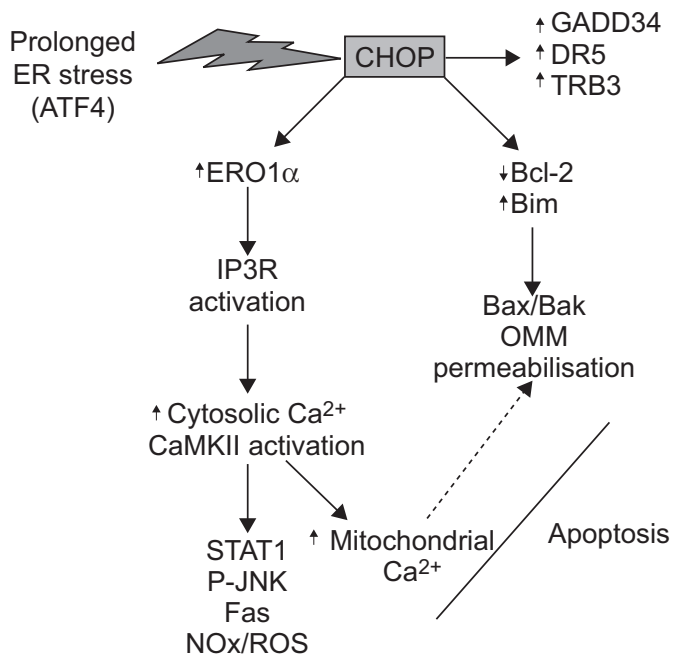

FIGURE 4. Maladaptive endoplasmic reticulum (ER) stress. This process generally helps the cell to survive, but if stress is overwhelming or prolonged, the cell will be driven to apoptosis. a) Prolonged activation of inositol-requiring protein (IRE)1 may promote apoptosis. b) Pathways through which prolonged activation of C/EBP homologous protein (CHOP) may promote apoptosis. XBP-1: X-box binding protein-1; TRAF2: tomour necrosis factor (TNF) receptor-associated factor 2; AIP1: ASK1interacting protein 1; ASK1: apoptosis signal-regulating kinase 1; P-JNK: phosphorylated c-Jun N-terminal kinase; Bax: apoptosis regulator Bax; Bak: Bcl-2 homologous antagonist/killer; RIDD: regulated IRE1 dependent decay; ATF-4: activating transcription factor 4; ERO1 $\alpha$ : ER oxidoreductin-1 $\alpha$; IP3R: inositol 1,4,5-triphosphate (Ip3) receptor; STAT: signal transducer and activator of transcription; Fas: TNF ligand superfamily member 6/Fas antigen ligand; NOx: nitric oxide; ROS: reactive oxygen species; GADD34: growth arrest and DNA damage-inducible protein GADD34; DR5: death receptor 5; TRB 3: Tribbles homolog 3; Bcl-2: B-cell lymphoma 2/apoptosis regulator Bcl-2; Bim: Bcl-2 like protein 11/Bcl2-interacting mediator of cell death; OMM: outer mitochondrial membrance. Reproduced from [33] with permission from the publisher. 


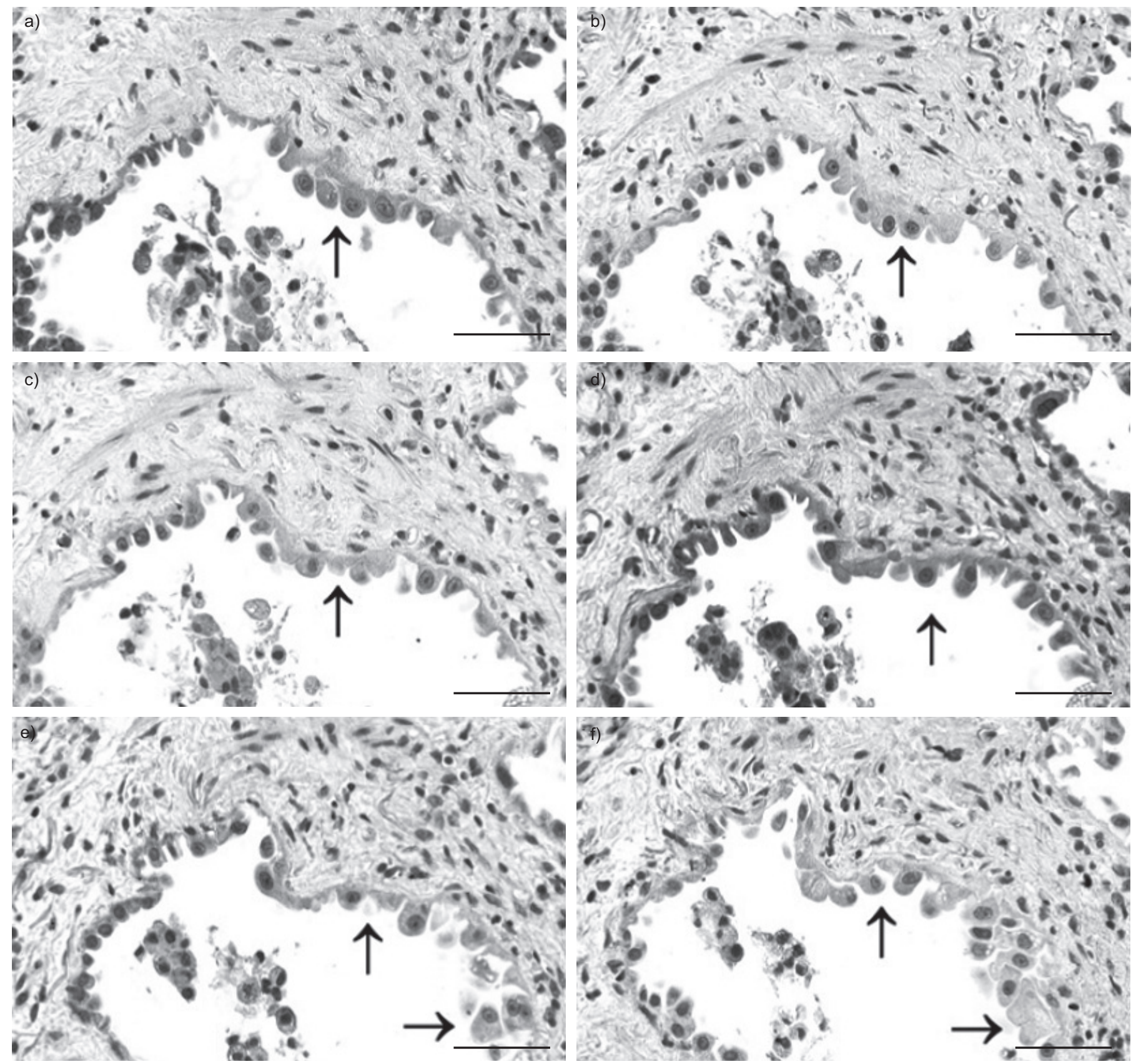

FIGURE 5. Hyperplastic alveolar epithelial type II cells show severe endoplasmic reticulum stress and consecutive apoptosis. Representative immunohistochemistry for a, d) pro-surfactant protein C, b) cleaved caspase 3, c) C/EBP homologous protein, e) activating transcription factor (ATF)-6 and f) ATF-4 in serial sections of idiopathic pulmonary fibrosis lung tissue. Scale bars $=50 \mu \mathrm{m}$. Reproduced from [10] with permission from the publisher.

in the carboxyl-terminal part of SP-C [26, 31]. Due to its interaction with the surfactant lipids, SP-C is a very hydrophobic protein. If mature SP-C were expressed in AECIIs, the result would be immediate cell death, as this protein would attack the cell. To avoid this problem, the SP-C protein produced inside the AECII initially incorporates "pro-protein" parts on both the amino- and carboxyl-terminal ends, which are successively cleaved during the passage and transport of the protein through the lysosomal compartment of AECIIs. At the end of this process, the mature SP-C protein is co-secreted with the lipids comprising surfactant (fig. 2).
However, in the case of the mutated form of SP-C, the carboxyl-terminus is altered in structure and misfolded. Due to this misfolding, the mutated SP-C pro-protein cannot be further processed by the AECII and, thus, accumulates in the cell and appears to co-aggregate with the healthy SP-C. This deposition of unfolded aggregated surfactant proteins seems to cause chronic injury to the AECII (fig. 2) [26].

\section{ER STRESS CAN BE PROTECTIVE AND DESTRUCTIVE}

It is important to understand the mechanism of ER stress, which is usually a very cytoprotective mechanism (fig. 3). The 

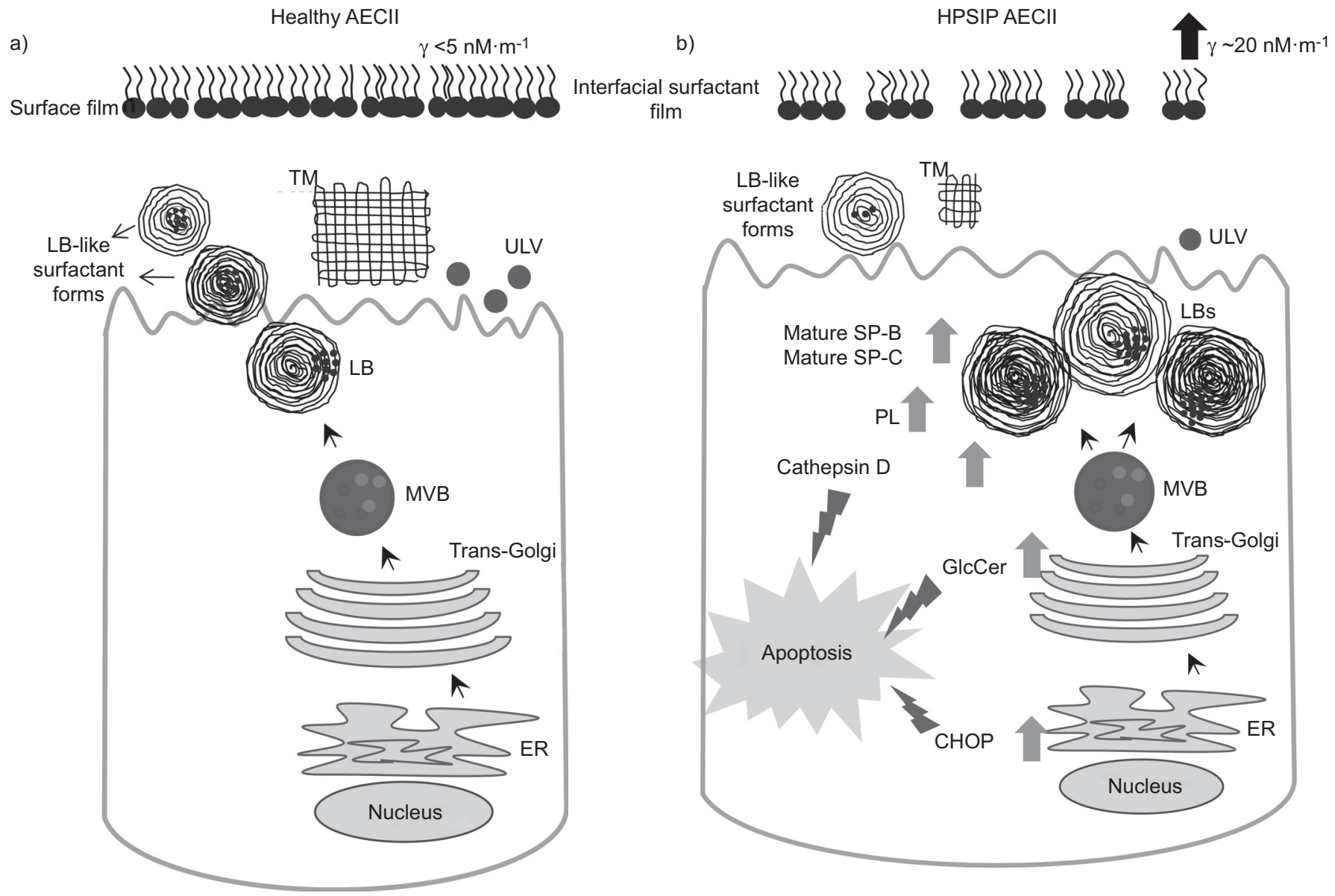

FIGURE 6. a) Healthy alveolar epithelial cells type II (AECII) and b) Hermansky-Pudlak syndrome-associated interstitial pneumonia (HPSIP) AECII. Disturbed intracellular surfactant transport in AECII occurs. The mature forms of surfactant proteins are blocked inside the late lysosomal compartment of the cell, but not primarily in the endoplasmic reticulum (ER) or Trans-golgi. The AEClls become swollen due to the accumulation of the mature surfactant proteins, which in turn causes lysosomal stress reactions through activation of cathepsin D, glycerol ceramides and eventually C/EBP homologous protein (CHOP). GICcer: glucosylceramides; LB: lamellar bodies; MVB: multivesicular bodies; PL: phospholipids; SP: surfactant protein; TM: tubular myelin; ULV: unilamellar vesicles.

ER contains chaperones that help protein folding, of which the most important is BiP, along with three other key signalling molecules: IRE1, ATF6 p90 and PERK. BiP usually interacts with these three key signalling molecules, keeping them in an inactivated state. If $\mathrm{BiP}$ is required to dissociate from the signalling molecules, to help client proteins to fold, IRE1, ATF6 p90 and PERK are activated and cause a redundant signalling process in the cell, with the primary purpose of aiding the cell to improve the folding of any misfolded proteins and to attain homeostasis again [32]. These 'redundant' signalling processes involve pathways that eventually affect lipid synthesis, overexpression of chaperones and compounds of the ER-associated protein degradation pathway (mainly proteasomal compounds), anti-reactive oxygen species signalling and arachidonic acid metabolism. This ER stress reaction generally helps the cell to survive; however, if the stress condition is overwhelming or prolonged, the cell will be driven to apoptosis via the activation of the two main factors; CHOP and ATF-4. ER stress may help the cell to survive or, under prolonged conditions, can drive the cell to apoptosis (fig. 4) [33].

\section{PATHOMECHANISMS OF SP-C MUTATIONS}

BRIDGES and co-workers [34, 35] examined the comparative effect of wild-type SP-C and mutated SP-C proteins on cell conditions.
HEK-293 cells were transfected to express either wild-type SP-C or mutated SP-C. The wild-type SP-C was found to co-localise in the lysosomal compartment, whereas mutated SP-C co-localise in the ER. It was demonstrated that a significant increase in ERstress reaction occurred within the cells expressing mutated SP-C. Subsequent secondary injury of the cells, through infection with a virus or blockage of proteasomal function, showed that the cells with mutated SP-C rapidly underwent apoptosis, whereas the cells with wild-type SP-C were not driven to cell death.

The clinical relevance of these observations is that respiratory infections tend to occur easily and may explain why many patients report infection prior to the exacerbation of IPF.

\section{SPORADIC IPF}

As with familial forms of IPF, a significant ER stress reaction driving the AECII to apoptosis is also seen in sporadic IPF and may be related to disturbed processing of surfactant compounds (fig. 5). Along with these intracellular changes, the composition of the alveolar surfactant pool is greatly altered and, thus, the surface tension of the alveolus is increased. As a result, higher pressure is needed to inflate the lungs; therefore, compliance is reduced, probably resulting in stretching of the distal lung [10]. 


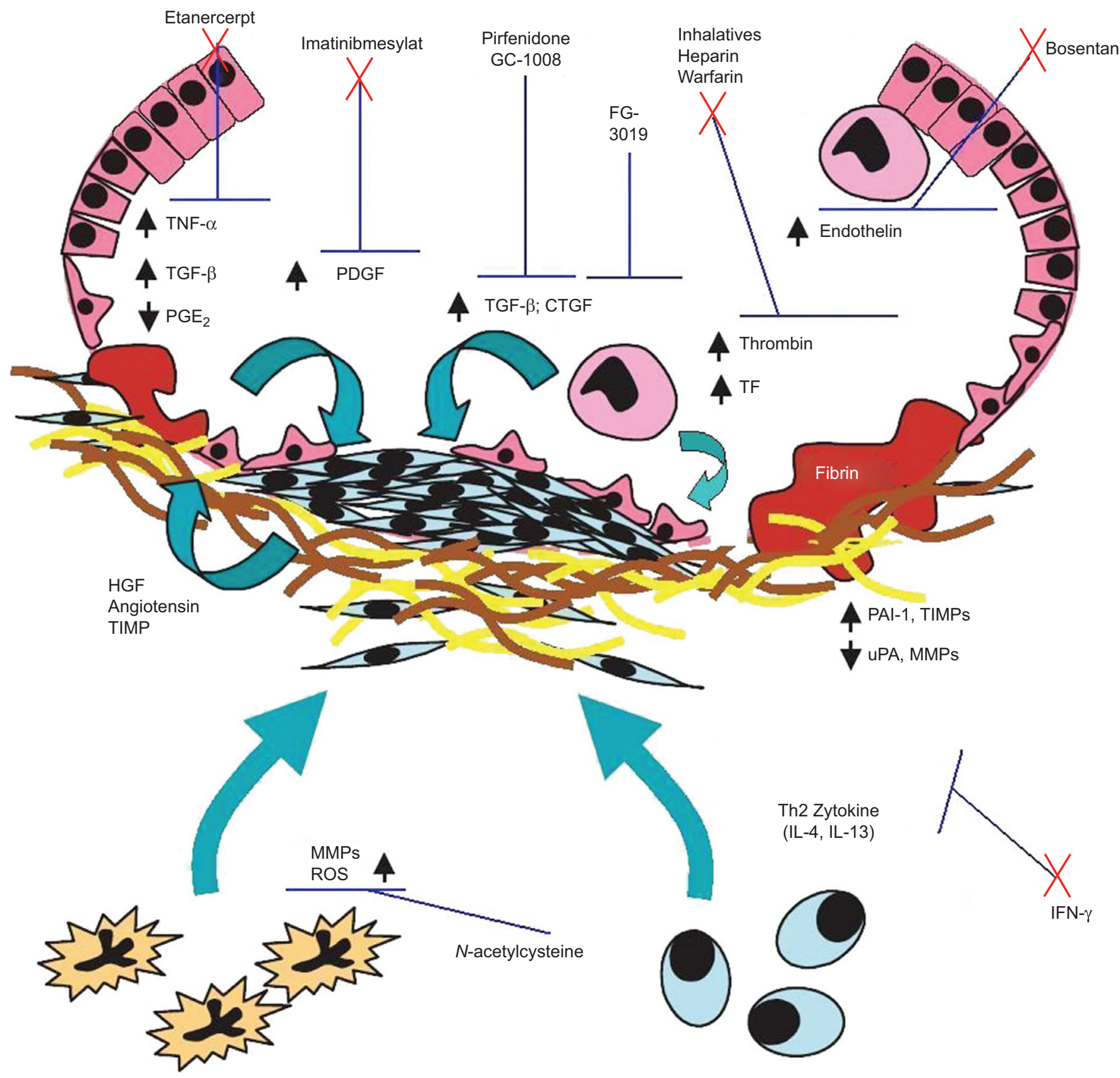

FIGURE 7. Current therapeutic approaches targeting profibrotic signalling pathways. Those pathways potentially leading to fibrosis have been used to develop targeted therapies for idiopathic pulmonary fibrosis treatment. Some potential therapies, which have had success in other disease areas, have failed. For example, tumour necrosis factor (TNF)- $\alpha$ blockers (e.g. etanercept) have been shown to be ineffective, and it appears that there is no significant role for agents that act on the endothelin pathway (e.g. bosentan). Therapies targeting anti-fibrotic and growth factor pathways are currently being developed. TGF- $\beta$ : transforming growth factor- $\beta$; PGE 2 : prostaglandin $E_{2}$; PDGF platelet-derived growth factor; CTGF: connective tissue growth factor; TF: transcription factor; HGF: hepatocyte growth factor; TIMP: tissue inhibitor of metalloproteinases; PAl-1: plasminogen activator inhibitor-1; uPA: urokinase-type plasminogen activator; MMP: matrix metalloproteinases; ROS: reactive oxygen species; TH2: T-helper 2 cell; IL: interleukin; IFN: interferon. Reproduced from [48] with permission from the publisher.

In IPF a key histological pattern that is observed is UIP, which may also be observed in other conditions. A rare example is Hermansky-Pudlak syndrome-associated interstitial pneumonia (HPSIP), which occurs in $\sim 40 \%$ of patients with HermanskyPudlak Syndrome (HPS); a lysosomal transport deficiency disease affecting the whole body. HPSIP has the same UIP pattern, histopathology and high-resolution computed tomography appearance as IPF. Therefore, the underlying molecular mechanism of this disease may help in the understanding of IPF. In fact, MAHVADI et al. [36] studied murine analogues of HPS and, surprisingly, again found that disturbances with regard to intracellular surfactant transport in AECII are involved [36]. In this case, the mature forms of SPs ready to be excreted are blocked inside the late lysosomal compartment of the cell but not 
primarily in the ER or trans-Golgi network. The AECIIs become swollen due to the accumulation of the mature SPs, which seems to cause a lysosomal stress reaction through activation of cathepsin D, glycerol ceramides and, eventually, CHOP (fig. 6).

\section{FROM AECII INJURY TO LUNG FIBROSIS}

There are three different theories under discussion regarding the mechanisms through which injury to the AECIIs results in lung fibrosis. The first involves epithelial-mesenchymal transition (EMT), with epithelial cells undergoing transdifferentiation into fibroblasts and consecutive activation. While this process has clearly been shown to occur in vivo in mice [37], there is still debate with regard to the importance of this pathway in IPF patients [38, 39]. According to a recent paper, not many mesenchymal cells seem to have originated from epithelial cells through EMT [39]. The second theory proposes that the dying AECII loses control over the mesenchymal cells and, as a result, the mesenchymal cells proliferate and produce more collagen. In detail, prostaglandin $(\mathrm{PG}) \mathrm{E}_{2}$ has been shown to be a key factor controlling fibroblast differentiation and proliferation [40]. In IPF, $\mathrm{PGE}_{2}$ levels are greatly reduced and, therefore, the absence of control of fibroblastic proliferation due to excessive epithelial apoptosis may be of importance. Moreover, it has been shown that chronically injured alveolar epithelial cells release a number of pro-fibrotic compounds, such as transforming growth factor- $\beta$, connective tissue growth factor, tissue factor, factor VII and factor $X$, that together activate mesenchymal cells [41-44]. A third putative mechanism may be that the dying AECII release factors such as stromal cell-derived factor-1 attracting circulating fibrocytes, which in turn invade the lung and locally expand the fibroblast pool [45]. In this regard, the number of circulating fibrocytes has been shown to be greatly increased in acute exacerbations of IPF [46].

Currently, it is not fully understood to what extent these proposed mechanisms actually play a part in the transition to lung fibrosis; however, each mechanism results in fibroproliferation, expansion of the fibroblast pool and the accumulation of collagen.

In terms of mediators of lung fibrosis, it is important to understand that different mechanisms lead to AECII apoptosis (ER stress via protein misfolding, DNA damage through telomerase shortening and lysosomal stress as seen in HPS) and that there are a multitude of different pathways that may result in fibrosis (for example pathways involving chemokines, pro-coagulant factors, anti-fibrinolytic factors, growth factors, leukotrienes and endothelin). With regard to the pathways potentially leading to fibrosis, these have been used to develop targeted therapies for the treatment of IPF (fig. 7) [47, 48]. Some of the potential therapies previously studied, and which have had success in other disease areas, have failed. For example, tumour necrosis factor- $\alpha$ blockers (e.g. etanercept) have been shown to be ineffective, and it appears that there is no significant role for agents that act on the endothelin pathway (e.g. bosentan) [49]. Therapies targeting the antifibrotic and growth factor pathways are currently being developed.

\section{TARGETING THE ANTI-FIBROTIC PATHWAY IN PATIENTS WITH IPF}

Pirfenidone is an orally available molecule that has demonstrated both anti-fibrotic and anti-inflammatory activity in vitro and in vivo. It appears to decrease fibroblast proliferation and reduce collagen formation and has been shown to be effective in different in vivo models of organ fibrosis; not only the lung, but also in the heart and kidney [50,51].

In a pre-clinical mouse model of bleomycin-induced lung fibrosis, pirfenidone was shown to significantly reduce the extent of lung fibrosis, as well as reduce the number and magnitude of myofibroblast responses [52]. Indeed, the pre-clinical data show that pirfenidone exerts anti-fibrotic actions, and it has recently been demonstrated to be effective in clinical trials of patients with IPF [53-55]. The primary end-point of change in percentage predicted forced vital capacity was found to be significantly increased in one of the two larger randomised, placebo-controlled trials, and a recent Cochrane meta-analysis of the available data from pirfenidone studies suggested that treatment with pirfenidone appears to slow down, but not completely arrest, the natural course of the disease [56].

Moreover, data from a recent phase II trial employing a novel triple-kinase inhibitor (BIBF 1120) targeting the receptors of fibroblast growth factor, vascular endothelial growth factor and platelet-derived growth factor, suggest that this compound may be similarly effective in IPF, although this has yet to be proven in currently ongoing phase III trials [57].

\section{SUMMARY AND CONCLUSIONS}

As we increase the understanding of the pathophysiological mechanisms involved in IPF, it is clear that there is evidence linking chronic AECII injury to the development of UIP/IPF. AECII injury is primarily mediated through ER stress, lysosomal stress, and mitochondrial and DNA damage. Secondary injuries, due to factors such as smoking or infection, may also have an important role in the development of the disease.

There are multiple pathways for chronic AECII injury to convert to fibrosis and, thus, many potential targets for developing new treatments for IPF. The growth factor and anti-fibrotic pathways seem to be important current targets, as demonstrated with the anti-fibrotic pirfenidone, which has shown strong pre-clinical anti-fibrotic activity and has been shown to be effective in clinical trials of patients with IPF.

\section{STATEMENT OF INTEREST}

P. Markart has received fees for speaking from Roche and InterMune.

\section{REFERENCES}

1 American Thoracic Society, European Respiratory Society. American Thoracic Society/European Respiratory Society International Multidisciplinary Consensus Classification of the Idiopathic Interstitial Pneumonias. Am J Respir Crit Care Med 2002; 165: 277-304

2 Raghu G, Collard HR, Egan JJ, et al. An official ATS/ERS/JRS/ ALAT statement: idiopathic pulmonary fibrosis: evidence-based guidelines for diagnosis and management. Am J Respir Crit Care Med 2011; 183: 788-824.

3 Hodgson U, Laitinen T, Tukiainen P. Nationwide prevalence of sporadic and familial idiopathic pulmonary fibrosis: evidence of founder effect among multiplex families in Finland. Thorax 2002; 57: 338-342.

4 Orphanet Report Series - Prevalence of rare diseases. Bibliographic data. November 2011, Number 1. www.orpha.net/orphacom/cahiers/ docs/GB/Prevalence_of_rare_diseases_by_alphabetical_list.pdf 
5 Vancheri C, Failla M, Crimi N, et al. Idiopathic pulmonary fibrosis: a disease with similarities and links to cancer biology. Eur Respir J 2010; 35: 496-504.

6 Myers JL, Katzenstein AL. Epithelial necrosis and alveolar collapse in the pathogenesis of usual interstitial pneumonia. Chest 1988; 94 1309-1311.

7 Selman M, King TE Jr, Pardo A. Idiopathic pulmonary fibrosis. Prevailing and evolving hypothesis about its pathogenesis and implications for therapy. Ann Intern Med 2001; 134: 136-151.

8 Selman M, Pardo A. Role of epithelial cells in idiopathic pulmonary fibrosis. From innocent targets to serial killers. Proc Am Thorac Soc 2006; 3: 364-372.

9 Du Bois RM. Strategies for treating idiopathic pulmonary fibrosis. Nat Rev Drug Discov 2010; 9: 129-140.

10 Korfei M, Ruppert L, Mahavadi P, et al. Epithelial endoplasmic reticulum stress and apoptosis in sporadic idiopathic pulmonary fibrosis. Am J Respir Crit Care Med 2008; 178: 838-846.

11 Pattle R. Properties, function and origin of the alveolar lining layer. Nature 1955; 175: 1125-1126.

12 Clements JA. Surface tension of lung extracts. Proc Soc Exp Biol Med 1957; 95: 170-172.

13 Tierney DF. Lung surfactant: some historical perspectives leading to its cellular and molecular biology. Am J Physiol 1989; 257: L1-L12.

14 Fehrenbach $\mathrm{H}$. Alveolar epithelial type II cell: defender of the alveolus revisited. Respir Res 2001; 2: 33-46.

15 Pison U, Max M, Neuendank A, et al. Host defence capacities of pulmonary surfactant: evidence for "non-surfactant" functions of the surfactant system. Eur J Clin Invest 1994; 24: 586-599.

16 Wright JR. Host defence functions of surfactant. In: Rooney SA, ed. Lung Surfactant: Cellular and Molecular Processing. Austin, R.G. Landes Company, 1998; pp. 191-214.

17 Crouch EC. Surfactant protein-D and pulmonary host defence. Respir Res 2000; 1: 93-108.

18 Singh G, Katyal SL, Brown WE, et al. Pulmonary lysozyme: a secretory protein of type II pneumocytes in the rat. Am Rev Respir Dis 1988; 138: 1261-1267.

19 Haller EM, Shelley SA, Montgomery MR, et al. Immunocytochemical localization of lysozyme and surfactant protein A in rat type II cells and extracellular surfactant forms. I Histochem Cytochem 1992; 40: 1491-1500.

20 Daniels CB, Lopatko OV, Orgeig S. Evolution of surface activity related functions of vertebrate pulmonary surfactant. Clin Exp Pharmacol Physiol 1998; 25: 716-721.

21 Hills BA. An alternative view of the role(s) of surfactant and the alveolar model. J Appl Physiol 1999; 87: 1567-1583.

22 Kauffman SL. Cell proliferation in the mammalian lung. Int Rev Exp Pathol 1980; 22: 131-191.

23 Uhal BD. Cell cycle kinetics in the alveolar epithelium. Am J Physiol 1997; 272: L1031-L1045.

24 Bhaskaran M, Kolliputi N, Wang Y, et al. Trans-differentiation of alveolar epithelial type II cells to type I cells involves autocrine signaling by transforming growth factor- $\beta 1$ through the Smad pathway. J Biol Chem 2007; 282: 3968-3976.

25 Thomas AQ, Lane K, Phillips J 3rd, et al. Heterozygosity for a surfactant protein $C$ gene mutation associated with usual interstitial pneumonitis and cellular nonspecific interstitial pneumonitis in one kindred. Am J Respir Crit Care Med 2002; 165 1322-1328.

26 Nogee LM, Dunbar AE 3rd., Wert SE., et al. A mutation in the surfactant protein $C$ gene associated with familial interstitial lung disease. N Engl J Med 2001; 344: 573-579.

27 Nogee LM, Dunbar AE 3rd., Wert SE., et al. Mutations in the surfactant protein $\mathrm{C}$ gene associated with interstitial lung disease. Chest 2002; 121: Suppl. 3, 20S-21S.

28 Cameron HS, Somaschini M, Carrera P, et al. A common mutation in the surfactant protein $C$ gene associated with lung disease. J Pediatr 2005; 146: 370-375.
29 Brasch F, Griese M, Tredano M, et al. Interstitial lung disease in a baby with a de novo mutation in the SFTPC gene. Eur Respir J 2004; 24: 30-39.

30 Alder JK, Chen JJ, Lancaster L, et al. Short telomeres are a risk factor for idiopathic pulmonary fibrosis. Proc Natl Acad Sci USA 2008; 105: 13051-13056.

31 Beers MF, Mulugeta S. Surfactant protein C. Biosynthesis and its emerging role in conformational lung disease. Annu Rev Physiol 2005; 67: 663-696.

32 Zhang K, Kaufman RJ. Signaling the unfolded protein response from the endoplasmic reticulum. J Biol Chem 2004; 279: 25936-25938.

33 Tabas I, Ron D. Integrating the mechanisms of apoptosis induced by endoplasmic reticulum stress. Nature Cell Biol 2011; 13: 184-190.

34 Bridges JP, Wert SE, Nogee LM, et al. Expression of a human protein $\mathrm{C}$ mutation associated with interstitial lung disease disrupts lung development in transgenic mice. J Biol Chem 2003; 278: 52739-52746.

35 Bridges JP, Xu Y, Na CL, et al. Adaptation and increased susceptibility to infection associated with constitutive expression of misfolded SP-C. J Cell Biol 2006; 172: 395-407.

36 Mahavadi P, Korfei M, Henneke I, et al. Epithelial stress and apoptosis underlie Hermansky-Pudlak syndrome-associated interstitial pneumonia. Am J Respir Crit Care Med 2010; 182: 207-219.

37 Kim KK, Wei Y, Szekeres C, et al. Epithelial cell $\alpha 3 \beta 1$ integrin links beta-catenin and Smad signaling to promote myofibroblast formation and pulmonary fibrosis. J Clin Invest 2009; 119: 213-224.

38 Marmai C, Sutherland RE, Kim KK, et al. Alveolar epithelial cells express mesenchymal proteins in patients with idiopathic pulmonary fibrosis. Am J Physiol Lung Cell Mol Physiol 2011; 301: L71-L78.

39 Rock JR, Barkauskas CE, Cronce MJ, et al. Multiple stromal populations contribute to pulmonary fibrosis without evidence for epithelial to mesenchymal transition. Proc Natl Acad Sci USA 2011; 108: E1475-E1483.

40 Okunishi K, Sisson TH, Huang SK, et al. Plasmin overcomes resistance to prostaglandin E2 in fibrotic lung fibroblasts by reorganizing protein kinase A signaling. J Biol Chem 2011; 286: 32231-32243.

41 Scotton CJ, Krupiczojc MA, Königshoff M, et al. Increased local expression of coagulation factor $\mathrm{X}$ contributes to the fibrotic response in human and murine lung injury. J Clin Invest 2009; 119: 2550-2563.

42 Howell DC, Johns RH, Lasky JA, et al. Absence of proteinaseactivated receptor-1 signaling affords protection from bleomycininduced lung inflammation and fibrosis. Am J Pathol 2005; 166: 1353-1365.

43 Wygrecka M, Kwapiszewska G, Jablonska E, et al. Role of protease-activated receptor-2 in idiopathic pulmonary fibrosis. Am J Respir Crit Care Med 2011; 183: 1703-1714.

44 Horan GS, Wood S, Ona V, et al. Partial inhibition of integrin $\alpha(v) \beta 6$ prevents pulmonary fibrosis without exacerbating inflammation. Am J Respir Crit Care Med 2008; 177: 56-65.

45 Phillips RJ, Burdick MD, Hong K, et al. Circulating fibrocytes traffic to the lungs in response to CXCL12 and mediate fibrosis. J Clin Invest 2004; 114: 438-446.

46 Moeller A, Gilpin SE, Ask K, et al. Circulating fibrocytes are an indicator of poor prognosis in idiopathic pulmonary fibrosis. Am Respir Crit Care Med 2009; 179: 588-594.

47 Thannickal VJ, Toews GB, White ES, et al. Mechanisms of pulmonary fibrosis. Ann Rev Med 2004; 55: 395-417.

48 White ES, Lazar MH, Thannickal VJ. Pathogenic mechanisms in unusual interstial pneumonia/idiopathic pulmonary fibrosis J Pathol 2003; 201: 343-354.

49 Gomer RH, Lupher ML Jr. Investigational approaches to therapies for idiopathic pulmonary fibrosis. Expert Opin Investig Drugs 2010; 19: 737-745. 
50 Maher TM. Pirfenidone in idiopathic pulmonary fibrosis. Drugs Today 2010; 46: 473-482.

51 Jackson RM, Gomez-Martin O. Development and utility of pirfenidone in the treatment of idiopathic pulmonary fibrosis: review of preclinical science and recent clinical trials. Transplant Res Risk Manage 2011; 3: 55-63.

52 Kakugawa T, Mukae H, Hayashi T, et al. Pirfenidone attenuates expression of HSP47 in murine bleomycin-induced pulmonary fibrosis. Eur Respir J 2004; 24: 57-65.

53 Noble PW, Albera C, Bradford WZ, et al. Pirfenidone in patients with idiopathic pulmonary fibrosis (CAPACITY): two randomised trials. Lancet 2011; 377: 1760-1769.
54 Taniguchi H, Ebina M, Kondoh Y, et al. Pirfenidone in idiopathic pulmonary fibrosis. Eur Respir J 2010; 35: 821-829.

55 Azuma A, Nukiwa T, Tsuboi E, et al. Double-blind, placebo-controlled trial of pirfenidone in patients with idiopathic pulmonary fibrosis. Am J Respir Crit Care Med 2005; 171: 1040-1047.

56 Spagnolo P, Del Giovane C, Luppi F, et al. Non-steroid agents for idiopathic pulmonary fibrosis. Cochrane Database Syst Rev 2010; 9: CD003134.

57 Richeldi L, Costabel U, Selman M, et al. Efficacy of a tyrosine kinase inhibitor in idiopathic pulmonary fibrosis. $N$ Engl J Med 2011; 365: 1079-1087. 